\title{
Prognostic Value of Tumor Regression Grade on MR in Rectal Cancer: A Large-Scale, Single-Center Experience
}

\author{
Heera Yoen, $M D^{1 *}$, Hye Eun Park, $M D^{2 * \dagger}$, Se Hyung Kim, $M D^{1,3,4}$, Jeong Hee Yoon, $M D^{1,3}$, Bo Yun Hur, MD ${ }^{5}$, \\ Jae Seok Bae, $M D^{1,3}$, Jung Ho Kim, $M D^{2}$, Hyeon Jeong Oh, $M D^{2}$, Joon Koo Han, $M D^{1,3,4}$ \\ Departments of ${ }^{1}$ Radiology and ${ }^{2}$ Pathology, Seoul National University Hospital, Seoul, Korea; ${ }^{3}$ Department of Radiology, Seoul National University \\ College of Medicine, Seoul, Korea; ${ }^{4}$ Institute of Radiation Medicine, Seoul National University Medical Research Center, Seoul, Korea; ${ }^{5}$ Department \\ of Radiology, Healthcare System Gangnam Center, Seoul National University Hospital, Seoul, Korea
}

Objective: To determine the prognostic value of MRI-based tumor regression grading (mrTRG) in rectal cancer compared with pathological tumor regression grading (pTRG), and to assess the effect of diffusion-weighted imaging (DWI) on interobserver agreement for evaluating mrTRG.

Materials and Methods: Between 2007 and 2016, we retrospectively enrolled 321 patients (male:female = 208:113; mean age, 60.2 years) with rectal cancer who underwent both pre-chemoradiotherapy (CRT) and post-CRT MRI. Two radiologists independently determined mrTRG using a 5-point grading system with and without DWI in a one-month interval. Two pathologists graded pTRG using a 5-point grading system in consensus. Kaplan-Meier estimation and Cox-proportional hazard models were used for survival analysis. Cohen's kappa analysis was used to determine interobserver agreement.

Results: According to mrTRG on MRI with DWI, there were $6 \mathrm{mrTRG}$ 1, $48 \mathrm{mrTRG} 2,109 \mathrm{mrTRG}$ 3, $152 \mathrm{mrTRG}$ 4, and 6 mrTRG 5. By pTRG, there were 7 pTRG 1, 59 pTRG 2, 180 pTRG 3, 73 pTRG 4, and 2 pTRG 5. A 5-year overall survival (OS) was significantly different according to the 5 -point grading $\operatorname{mrTRG}(p=0.024)$ and $\mathrm{pTRG}(p=0.038)$. The 5 -year disease-free survival (DFS) was significantly different among the five mrTRG groups $(p=0.039)$, but not among the five pTRG groups $(p=0.072)$. OS and DFS were significantly different according to post-CRT MR variables: extramural venous invasion after CRT (hazard ratio $=2.259$ for 0 S, hazard ratio $=5.011$ for DFS) and extramesorectal lymph node (hazard ratio $=2.610$ for DFS). For mrTRG, $\mathrm{k}$ value between the two radiologists was 0.309 (fair agreement) without DWI and slightly improved to 0.376 with DWI. Conclusion: mrTRG may predict OS and DFS comparably or even better compared to PTRG. The addition of DWI on T2-weighted MRI may improve interobserver agreement on mrTRG.

Keywords: Rectal neoplasm; Regression; Magnetic resonance imaging; Prognosis; Survival analysis

\section{INTRODUCTION}

For locally invasive rectal cancer, the standard treatment has been neoadjuvant chemoradiotherapy (CRT) followed by total mesorectal excision, which improves curative results (1).
After surgical resection, pathological staging of the tumor is assessed for tumor TNM stage according to extramural extension, regional nodal status, and involvement of the adjacent organs. It is suggested that pathological $\mathrm{T}$ and $\mathrm{N}$ category can be predictable features for oncologic outcomes

Received: October 25, 2019 Revised: March 3, 2020 Accepted: March 12, 2020

This research was supported by the Basic Science Research Program of the National Research Foundation of Korea [NRF] funded by the Ministry of Science, ICT \& Future Planning (NRF-2019R1F1A1060131) and from the Seoul National University Hospital Research Fund No. 04-2020-2010.

*These authors contributed equally to this work.

${ }^{\dagger}$ Current affiliation: Department of Pathology, Seoul Metropolitan Government Seoul National University Boramae Medical Center, Seoul, Korea Corresponding author: Se Hyung Kim, MD, Department of Radiology, Seoul National University Hospital, 101 Daehak-ro, Jongno-gu, Seoul 03080, Korea.

- E-mail: shkim7071@gmail.com

This is an 0pen Access article distributed under the terms of the Creative Commons Attribution Non-Commercial License (https:// creativecommons.org/licenses/by-nc/4.0) which permits unrestricted non-commercial use, distribution, and reproduction in any medium, provided the original work is properly cited. 
such as local recurrence, disease-free survival (DFS), and overall survival (OS) (2). However, several recent studies have reported that the oncologic outcomes are better correlated with pathological evaluation of the neoadjuvant CRT response (3-5). Qualitative evaluation using tumor regression grade (TRG) to evaluate tumor cell replaced by fibrosis in response to CRT was suggested as a good parameter, and it has been widely accepted as a practical parameter for oncologic prognosis in rectal cancer patients (6-9).

Accurate preoperative assessment of response to therapy may permit the clinicians to modify definitive treatment (10). A number of different methods have been proposed in assessing response of rectal cancer to CRT on MRI. They include posttreatment T and N category on MRI (ymrTN), volume reduction between baseline and posttreatment, and modified Response Evaluation Criteria in Solid Tumors measurement (10-14). In addition to these assessment parameters, the Magnetic Resonance Imaging and Rectal Cancer European Equivalence (MERCURY) study group proposed an MRI-based tumor regression grading (mrTRG) system by applying the principles of pathological tumor regression grading (pTRG) system (15). They evaluated mrTRG after neoadjuvant CRT by discriminating viable tumor signals from fibrosis on MRI's, and showed its predictive value for survival outcomes $(10,16,17)$. However, there has been insufficient evidence for comparison between mrTRG and pTRG on overall patients' prognosis (18).

Diffusion-weighted imaging (DWI) and resulting apparent diffusion coefficient (ADC) values have also been used to characterize tumors and to quantify treatment-induced changes because viable tumor components can be easily discriminated from fibrosis by addition of DWI $(19,20)$. Indeed, several prior studies suggested an added diagnostic value of DWI to conventional T2-weighted images (T2WIs), and the changes of ADC after CRT in rectal cancer are also known to have prognostic and predictive implications (1922). However, there has been insufficient evidence on interobserver agreement for DWI in predicting treatment response evaluation for locally advanced rectal cancer.

Therefore, this study aimed to determine the prognostic value of mrTRG in rectal cancer compared to PTRG. We also tried to assess the effect of DWI on interobserver agreement in assessing mrTRG.

\section{MATERIALS AND METHODS}

This retrospective study was approved by the Institutional
Review Board and research ethics committee of Seoul National University Hospital. The written informed consent form was waived.

\section{Patients}

Between January 2007 and December 2016, 500 patients who underwent both neoadjuvant CRT and surgery in our hospital were initially included. Among them, 35 patients were excluded due to insufficient pre-operative or postoperative MRI $(n=9)$, poor MR image quality $(n=2)$, refused in-time operation $(n=2)$, and patients with distant metastasis $(n=22)$. Among the remaining 465 patients with adequate pre-CRT and post-CRT MRI, 144 patients were further excluded due to unavailable pathologic slides for re-evaluating PTRG $(n=106)$ or due to the absence of DWI sequences in MRI $(n=51)$. Finally, 321 patients were enrolled for further analysis (Fig. 1). Information regarding patients' treatment and follow-up are described in detail in the Supplementary Materials.

\section{MR Acquisition}

All patients underwent both pre-CRT and post-CRT MRI using the $1.5 \mathrm{~T}$ or $3 \mathrm{~T}$ MR system. Post-CRT MRI was usually scheduled within 1 or 2 weeks (mean \pm standard deviation, $5.2 \pm 6.8$ days) before surgery and at $48.3 \pm 24.2$ days after completion of neoadjuvant CRT. Duration of CRT was $40.4 \pm$ 4.5 days. Detailed MR acquisition parameters are described in Table of Supplementary Materials.

\section{MR Image Analysis}

Two radiologists (with 10 and 3 years of experience

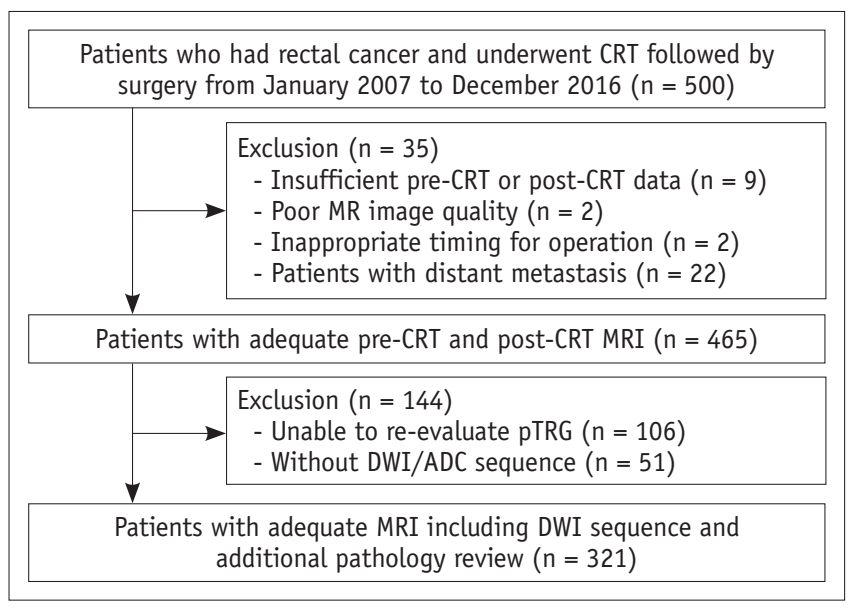

Fig. 1. Flow chart of patient population. $A D C=$ apparent diffusion coefficient, CRT = chemoradiotherapy, DWI = diffusion-weighted imaging, $\mathrm{pTRG}=$ pathological tumor regression grading 
in rectal MRI, respectively) assessed the T category, $\mathrm{N}$ category, extramesorectal lymph node (LN) status, extramural depth (EMD, mm), distance from mesorectal fascia ( $\mathrm{mm}$ ), and extramural venous invasion (EMVI) in consensus. By comparing pre- and post-CRT MRI, overall mrTRG was determined considering a proportion of fibrosis on post-CRT MRI according to a previously reported 5-point grading system: grade 1, no evidence of treated tumor; grade 2, dense hypointense fibrosis with minimal residual tumor; grade $3,>50 \%$ fibrosis or mucin with intermediate tumor signal representing residual tumor (fibrosis $>$ tumor); grade 4, minimal fibrosis or mucinous degeneration, but mostly tumor (tumor > fibrosis); and grade 5, tumor that has similar appearance as baseline (10).

Two other radiologists (with 8 and 6 years of experience in rectal MRI, respectively) additionally and independently reviewed the pre- and post-CRT MR images and evaluated mrTRG using the same 5-point grading system without knowing patients' clinical and pathologic information. MR image interpretation was performed first with highresolution axial, sagittal, and coronal T2WIs alone. mrTRG was determined by judging the proportion of the remaining intermediate high tumor signal and dark fibrosis on T2WIs. After 1 month, DWIs were additionally provided to assess the effect of DWI on interobserver agreement for mrTRG. Residual tumors were identified on DWI and ADC maps where the higher signal intensity (SI) remained compared to the adjacent normal rectal wall using high b-value DWI, with the corresponding low intensity appearing on the $A D C$ map.

\section{Histopathologic Analysis}

After total mesorectal excision, fresh specimens were obtained and fixed in $10 \%$ formalin solution and then prepared according to a previous study by Quirke et al. (23, 24). Resection margin, LN involvement, venous invasion, and invasion to other organs were evaluated.

For 321 patients who have available histopathologic slides, PTRG was re-evaluated by two pathologists (with 4 and 5 years of experience in rectal cancer pathology, respectively) independently using a 5-point grading system proposed by Mandard et al. (25). The pTRG system was described in the Supplementary Materials. If there was a discrepancy between the two pathologists, the third senior pathologist (with 8 years of experience in rectal cancer histopathology) reviewed the slides and ultimately reached a consensus.

\section{Statistical Analysis}

Cohen's kappa analysis was used to compare PTRG and mrTRG as well as to determine interobserver agreement between the two independent radiologists. Interpretation of kappa values is described in the Supplementary Materials. We further reclassified the patients into two groups (the good vs. poor response groups) according to tumor response; patients with TRG of 1 to 3 were classified as good responders, and TRG of 4 to 5 were classified as poor responders. We also performed dichotomous statistical analyses for these two groups. Tumors were also classified into two groups (good and poor responders) according to ypT category. ypT1, ypT2, and ypT3a were considered good responders, while ypT3b-3d and ypT4 were considered poor responders $(15,26)$.

Survival analysis was performed using the Kaplan-Meier method with a log-rank test. By using Cox proportional hazard regression methods, univariate and multivariate analyses were performed for variables in clinical (neoadjuvant chemotherapeutic agents, surgery type, and adjuvant treatment), pre- and post-CRT MRI findings with $O S$ and DFS. The variables that showed statistical significances in the univariate analyses were further included in the multivariate analysis to validate their statistical significances.

A $p$ value less than 0.05 was considered statistically significant. All statistical analyses were performed using SPSS version 25 software (IBM Corp., Armonk, NY, USA) and MedCalc statistical software version 18.6 (MedCalc Software, Ostend, Belgium).

\section{RESULTS}

\section{Patients' Demographics and Tumor Characteristics on MRI and Histopathology \\ Table 1 summarizes patients' demographic and clinical information. Table 2 shows tumor characteristics on pre- and post-CRT MRI and on the histopathology of all 321 patients.}

\section{Comparison between mrTRG and pTRG System}

Table 3 demonstrates the overall distribution of mrTRG and pTRG. Based on a 5-point grading system, the weighted kappa value between mrTRG and pTRG was 0.280 (fair agreement). Concordance between mrTRG and PTRG was observed in 143 patients $(44.5 \%, 143 / 321)$. Fortyseven patients $(26.4 \%, 47 / 178)$ were underestimated 
Table 1. Patients' Demographic and Clinical Information $(n=$ 321)

\begin{tabular}{|c|c|}
\hline Characteristics & Value \\
\hline \multicolumn{2}{|l|}{ Age (years) } \\
\hline Mean \pm SD & $60.2 \pm 11.1$ \\
\hline Range & $28-86$ \\
\hline \multicolumn{2}{|l|}{ Sex } \\
\hline Male & 208 \\
\hline Female & 113 \\
\hline \multicolumn{2}{|l|}{ Neoadjuvant treatment $(n=309) *$} \\
\hline 5-fluorouracil & 133 \\
\hline Capecitabine & 137 \\
\hline 5-fluorouracil + leucovorin & 31 \\
\hline FOLFOX ${ }^{\dagger}$ & 5 \\
\hline Radiotherapy alone & 3 \\
\hline \multicolumn{2}{|l|}{ Operation } \\
\hline APR & 25 \\
\hline LAR & 187 \\
\hline ULAR & 106 \\
\hline Transanal excision & 1 \\
\hline Hartmann's operation & 2 \\
\hline \multicolumn{2}{|l|}{ Adjuvant treatment } \\
\hline Yes & 269 \\
\hline No & 52 \\
\hline \multicolumn{2}{|c|}{ Time interval between CRT and post-CRT MRI } \\
\hline Mean \pm SD (days) & $5.2 \pm 6.8$ \\
\hline \multicolumn{2}{|c|}{ Time interval between post-CRT MRI to operation } \\
\hline Mean \pm SD (days) & $48.3 \pm 24.2$ \\
\hline
\end{tabular}

*Twelve patients had no available information regarding chemotherapy regimen because they received preoperative CRT at outside hospital, ${ }^{\dagger}$ FOLFOX regimen consists of three drugs of folinic acid (leucovorin), 5-fluorouracil, and oxaliplatin (eloxatin). $A P R=$ adominoperineal resection, $C R T=$ chemoradiotherapy, $L A R=$ low anterior resection, $\mathrm{SD}=$ standard deviation, ULAR $=$ ultra-low anterior resection

for tumor regression with mrTRG, while 131 patients (73.6\%, 131/178) were overestimated on MRI compared to pTRG. Using a binomial classification into good and poor responders, concordance rate was increased to $62.3 \%$ (200/321). However, the kappa value was still in fair agreement (kappa value $=0.240$ ) between mrTRG and pTRG. Representative examples of concordant and discordant cases between mrTRG and pTRG are presented in Figures 2 and 3.

\section{Effect of DWI on mrTRG Interpretation}

Based on a 5-point grading system, interobserver agreement for mrTRG was fair $(\kappa=0.309)$ between the two readers without DWI. It was slightly improved when DWI was added $(\kappa=0.376)$ (Table 4$)$. Based on a binomial classification, the weighted kappa value was also improved from 0.364 to 0.438 between the two reviewers with an addition of a DWI sequence. Representative examples showing the usefulness of DWI for determining mrTRG are presented in Figures 4 and 5 .

Prognostic Value of Clinical, MRI, and Histopathologic Features

\section{Related to OS}

Mean OS of all 321 patients was $68.1 \pm 30.7$ months. OS was not significantly different in relation to different neoadjuvant regimens $(p=0.867)$, type of surgery $(p=$ $0.069)$, or adjuvant treatment $(p=0.141)$. Significant MR features related to patients' OS are summarized in Table 5. All univariate results of MRI variables related to $0 S$ are listed in Supplementary Table 1. Among the 321 patients, 49 patients died due to disease. On a univariate analysis, no features on the pre-CRT MRI remained related to OS. On post-CRT MRI, ycN category (hazard ratio [HR], 2.295; $p=$ 0.005), EMVI after CRT (EMVI post $_{\text {) }}(H R, 3.314 ; p=0.002)$, EMD after CRT $\left(E M D_{\text {post }}\right)(H R, 1.082 ; p=0.016)$, and mrTRG (HR, 6.165; $p=0.024$ for 5-point grading and HR, 2.172; $p=0.010$ for binomial classification) were significant variables related to $0 \mathrm{~S}$. On a multivariate analysis, only EMVI $_{\text {post }}(H R, 2.259 ; p=0.048)$ on post-CRT MRI remained as a statistically significant factor for 0 .

For the association between histopathologic features and OS, ypT (HR, 2.161; $p=0.007)$,ypN (HR, 2.472; $p=0.002)$, extramesorectal LN (HR, 10.381; $p<0.001)$, and EMVI (HR, $23.345 ; p=0.002$ ) were significant features related to $0 S$ on a univariate analysis. On a multivariate analysis, ypT $(H R, 2.179 ; p=0.008)$ and extramesorectal $L N$ involvement $(H R, 11.005 ; p<0.001)$ were significant factors associated with OS.

Based on a 5-point grading system, OS was significantly different among the mrTRG groups as well as PTRG groups. The 5 -year OS rate was $100 \%$ (no event) for mrTRG 1 , $92.7 \%$ for mrTRG 2, $89.6 \%$ for mrTRG 3, 80.1\% for mrTRG 4 , and $40.0 \%$ for mrTRG $5(p=0.024)$, respectively. The 5 -year $\mathrm{OS}$ rates were also significantly different among the five pTRG groups ( $85.7 \%$ for pTRG $1,97.8 \%$ for pTRG 2, $84.3 \%$ for pTRG $3,74.9 \%$ for pTRG 4 , and $100 \%$ for pTRG 5 , respectively) $(p=0.038)$. Using a binomial classification, OS was significantly different between the mrTRG groups and between the pTRG groups. For mrTRG, the 5 -year OS rate was $91.0 \%$ for good responders and $79.1 \%$ for poor responders $(p=0.010)$. For $\mathrm{PTRG}$, the 5 -year $0 \mathrm{~S}$ rate was 
$88.3 \%$ for good responders and $75.6 \%$ for poor responders $(p=0.024)$. Survival curves of the five mrTRG and PTRG groups in terms of OS are presented in Figure 6.

\section{Related to DFS}

Mean DFS of all 321 patients was $53.5 \pm 32.4$ months.
Significant MR features related to patients' DFS are summarized in Table 5. All univariate results of MRI variables related to DFS are listed in Supplementary Table 2. Among 321 patients without distant metastasis at the time of initial diagnosis, tumor recurred in 45 patients $(14.0 \%)$; there was local recurrence in 10 patients; distant metastasis in

Table 2. Tumor Characteristics on Pre- and Post-CRT MRI and Pathology

\begin{tabular}{|c|c|c|c|c|c|}
\hline \multicolumn{2}{|c|}{ Pre-CRT MRI } & \multicolumn{2}{|c|}{ Post-CRT MRI } & \multicolumn{2}{|c|}{ Pathology } \\
\hline \multirow[t]{3}{*}{ cT category } & & ycT category & & ypT category & \\
\hline & & T0 & 7 & T0 & 7 \\
\hline & & & & Tis & 9 \\
\hline $\mathrm{T} 1$ & 1 & $\mathrm{~T} 1$ & 4 & $\mathrm{~T} 1$ & 19 \\
\hline $\mathrm{T} 2$ & 71 & $\mathrm{~T} 2$ & 153 & $\mathrm{~T} 2$ & 115 \\
\hline T3a & 3 & T3a & 2 & T3a & 40 \\
\hline $\mathrm{T} 3 \mathrm{~b}$ & 118 & $\mathrm{~T} 3 \mathrm{~b}$ & 95 & $\mathrm{~T} 3 \mathrm{~b}$ & 83 \\
\hline $\mathrm{T} 3 \mathrm{c}$ & 68 & $T 3 c$ & 35 & T3c & 45 \\
\hline T3d & 12 & T3d & 1 & $\mathrm{~T} 3 \mathrm{~d}$ & - \\
\hline T4a & 17 & T4a & 6 & $\mathrm{~T} 4 \mathrm{a}$ & 1 \\
\hline T4b & 31 & $\mathrm{~T} 4 \mathrm{~b}$ & 18 & T4b & 2 \\
\hline $\mathrm{cN}$ category & & ycN category & & ypN category & \\
\hline Negative & 58 & Negative & 240 & Negative & 240 \\
\hline Positive & 263 & Positive & 81 & Positive & 81 \\
\hline Extramesorectal $\mathrm{LN}_{\text {pre }}$ & & Extramesorectal $\mathrm{LN}_{\text {post }}$ & & yp-extrameso & \\
\hline Negative & 234 & Negative & 285 & Negative & 307 \\
\hline Positive & 87 & Positive & 36 & Positive & 14 \\
\hline $\mathrm{EMD}_{\text {pre }}(\mathrm{mm})$ & & $\mathrm{EMD}_{\text {post }}(\mathrm{mm})$ & & & \\
\hline Mean, SD & $4.7,5.1$ & Mean, SD & $2.4,3.4$ & & \\
\hline Range & $0-36.6$ & Range & $0-19.2$ & & \\
\hline $\operatorname{MRF}_{\text {pre }}(\mathrm{mm})$ & & $\mathrm{MRF}_{\text {post }}(\mathrm{mm})$ & & & \\
\hline Mean, SD & $5.7,5.4$ & Mean, SD & $6.5,5.9$ & & \\
\hline Range & $0-24.3$ & Range & $0-26.5$ & & \\
\hline EMVI $_{\text {pre }}$ & & $\mathrm{EMVI}_{\text {post }}$ & & ypEMVI & \\
\hline Negative & 279 & Negative & 294 & Negative & 320 \\
\hline Positive & 42 & Positive & 27 & Positive & 1 \\
\hline
\end{tabular}

$E M D=$ extramural depth, $E M D_{\text {post }}=E M D$ after $C R T, E M D_{\text {pre }}=E M D$ beforer CRT, EMVI $=$ extramural venous invasion, $E M V I_{\text {post }}=E M V I$ after $C R T$, $E M V I_{\text {pre }}=E M V I$ before $C R T, L N=$ lymph node, $L N_{\text {post }}=L N$ after $C R T, L N_{\text {pre }}=L N$ beforer $C R T, M R F=$ mesorectal fascia, $M R F_{\text {post }}=M R F$ after $C R T$, $M R F_{\text {pre }}=M R F$ beforer $C R T$

Table 3. Comparison between mrTRG and PTRG

\begin{tabular}{|c|c|c|c|c|c|c|c|c|}
\hline & & \multicolumn{5}{|c|}{ mrTRG } & \multirow{2}{*}{\multicolumn{2}{|c|}{ Total }} \\
\hline \multirow{2}{*}{\multicolumn{2}{|c|}{ pTRG }} & 1 & 2 & 3 & 4 & 5 & & \\
\hline & & & Gooc & & & & & \\
\hline 1 & \multirow{3}{*}{ Good } & 1 & 4 & 1 & 1 & 0 & 7 & \\
\hline 2 & & 4 & 21 & 20 & 14 & 0 & 59 & 246 \\
\hline 3 & & 1 & 21 & 71 & 85 & 2 & 180 & \\
\hline 4 & \multirow{2}{*}{ Poor } & 0 & 2 & 17 & 50 & 4 & 73 & \multirow{2}{*}{75} \\
\hline 5 & & 0 & 0 & 0 & 2 & 0 & 2 & \\
\hline \multirow{2}{*}{\multicolumn{2}{|c|}{ Total }} & 6 & 48 & 109 & 152 & 6 & \multirow{2}{*}{\multicolumn{2}{|c|}{321}} \\
\hline & & & 163 & & & & & \\
\hline
\end{tabular}

mrTRG = MRI-based tumor regression grading, $\mathrm{pTRG}=$ pathological tumor regression grading 
33 patients; both local recurrence and distant metastasis in 2 patients. On a univariate analysis, EMVI before CRT (HR, 3.120; $p=0.001)$ on pre-CRT MRI, ycT category (HR, 2.148; $p=0.015)$, ycN category $(H R, 2.501 ; p=0.003)$, extramesorectal LN (HR, 3.273; $p=0.001), \mathrm{EMVI}_{\text {post }}(\mathrm{HR}$, $5.879 ; p<0.001), \mathrm{EMD}_{\text {post }}(\mathrm{HR}, 1.111 ; p=0.001)$, mrTRG
(HR, 7.974; $p=0.039$ for 5 -point grading and HR, 1.917; $p=0.036$ for binomial classification) on post-CRT MRI were significant variables related to DFS. On a multivariate analysis, extramesorectal LN (HR, 2.610; $p=0.008)$ and EMVI $_{\text {post }}(H R, 5.011 ; p<0.001)$ on post-CRT MRI were statistically significant for DFS.

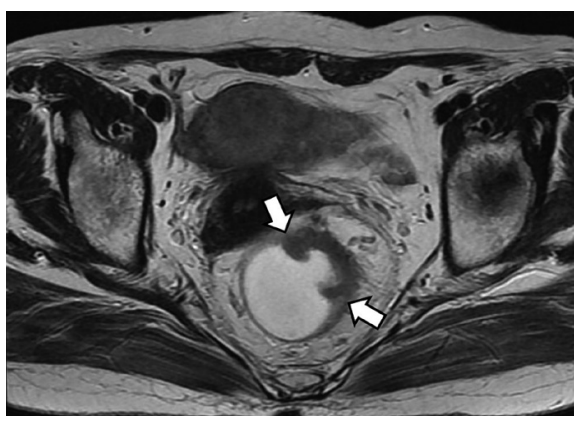

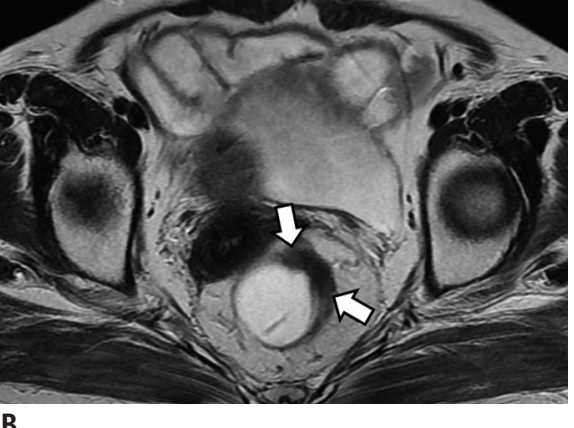

B

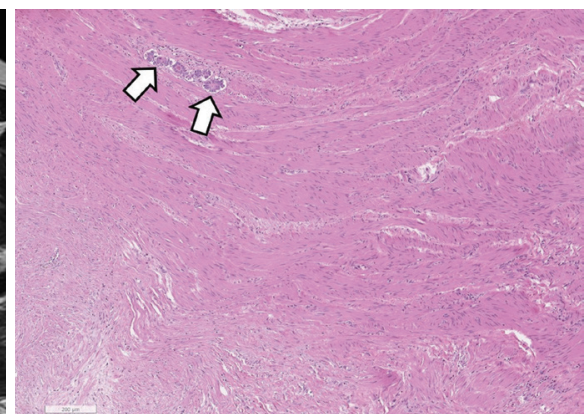

C

Fig. 2. Concordant case between mrTRG and pTRG in 60-year-old man with rectal cancer.

A. On T2W axial image before CRT, tumor (arrows) showing intermediate high SI is located between 12 and 3 o'clock on rectum. B. After CRT, most of tumor (arrows) was replaced by fibrosis with dark T2 SI. Both radiologists reported as mrTRG 2. C. Upon microscopic examination, most tumor cells are replaced by fibrosis and only scanty tumor cells (arrows) remain (H\&E stain; original magnification, $x$ 50). Therefore, pathologists graded pTRG as 2. H\&E = hematoxylin and eosin, mrTRG = MRI-based tumor regression grading, $\mathrm{SI}=$ signal intensity, T2W $=$ T2-weighted

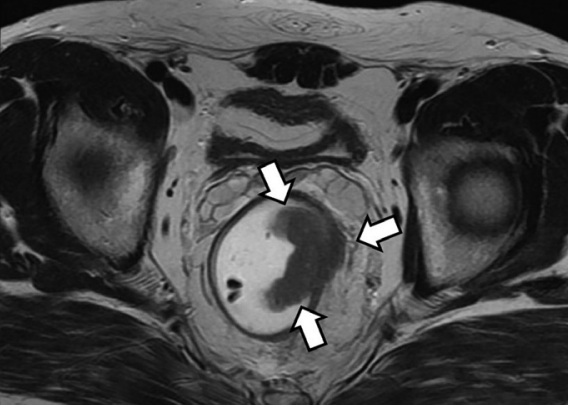

A

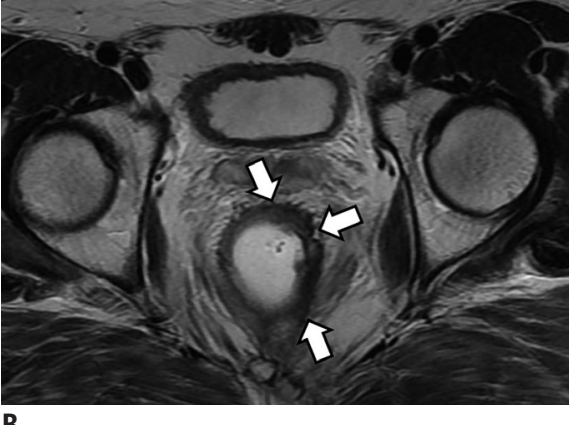

B

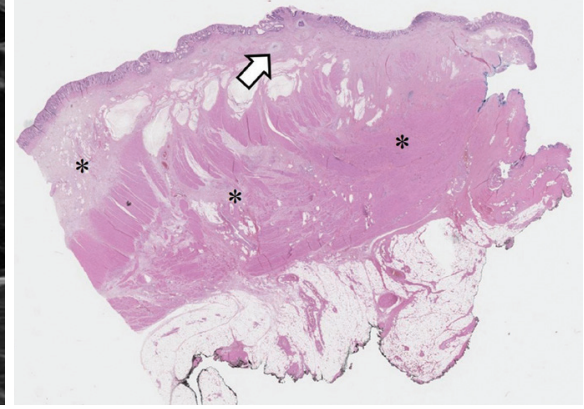

C

Fig. 3. Discordant case between mrTRG and pTRG in 48-year-old man with rectal cancer.

A. On T2W axial image before CRT, tumor (arrows) showing intermediate high SI is located between 1 and 4 o'clock on rectum. B. After CRT, most of tumor was replaced by dark fibrosis. However, there is remaining lesion (arrows) with intermediate high signal. Radiologists reported as mrTRG 3 (> 50\% fibrosis or mucin with intermediate tumor signal representing residual tumor, fibrosis $>$ tumor). C. However, on microscopic pathologic slides (H\&E stain; original magnification, $x$ 12.5), most tumor cells are replaced by fibrosis $\left({ }^{*}\right)$ and only scanty tumor cells (arrow) remain. Therefore, pathologists graded pTRG as 2 . This is case in which TRG was overestimated by MR compared to pathology due to CRT-related inflammation and edema. TRG = tumor regression grade

Table 4. Interobserver Agreement between Two Radiologists for mrTRG

\begin{tabular}{|c|c|c|c|c|c|c|c|c|c|c|c|c|}
\hline \multirow{3}{*}{ Reviewer 1} & \multicolumn{10}{|c|}{ Reviewer 2} & \multirow{2}{*}{\multicolumn{2}{|c|}{ Total (\%) }} \\
\hline & \multicolumn{2}{|c|}{ mrTRG 1} & \multicolumn{2}{|c|}{ mrTRG 2} & \multicolumn{2}{|c|}{ mrTRG 3} & \multicolumn{2}{|c|}{ mrTRG 4} & \multicolumn{2}{|c|}{ mrTRG 5} & & \\
\hline & $\begin{array}{l}\text { DWI (-) } \\
\text { DW }\end{array}$ & DWI (+) & DWI (-) & DWI $(+)$ & DWI (-) & DWI $(+)$ & $\begin{array}{l}\text { DWI }(-) \\
\text {. }\end{array}$ & DWI (+) & DWI (-) & DWI (+) & DWI (-) & DWI (+) \\
\hline mrTRG 1 & 0 & 0 & 0 & 0 & 0 & 1 & 0 & 0 & 0 & 0 & $0(0.0)$ & $1(0.3)$ \\
\hline mrTRG 2 & 1 & 2 & 11 & 8 & 37 & 34 & 4 & 5 & 0 & 0 & $53(16.5)$ & $49(15.3)$ \\
\hline mrTRG 3 & 3 & 2 & 11 & 2 & 64 & 78 & 41 & 32 & 0 & 0 & $119(37.1)$ & $114(35.5)$ \\
\hline $\operatorname{mrTRG} 4$ & 0 & 0 & 2 & 1 & 53 & 51 & 88 & 99 & 2 & 2 & $145(45.2)$ & $153(47.7)$ \\
\hline mrTRG 5 & 0 & 0 & 0 & 0 & 1 & 1 & 2 & 2 & 1 & 1 & $4(1.2)$ & $4(1.2)$ \\
\hline Total (\%) & $\begin{array}{c}4 \\
(1.2)\end{array}$ & $\begin{array}{c}4 \\
(1.2)\end{array}$ & $\begin{array}{c}24 \\
(7.5)\end{array}$ & $\begin{array}{c}11 \\
(3.4)\end{array}$ & $\begin{array}{c}155 \\
(48.3)\end{array}$ & $\begin{array}{c}165 \\
(51.4)\end{array}$ & $\begin{array}{c}135 \\
(42.1)\end{array}$ & $\begin{array}{c}138 \\
(43.0)\end{array}$ & $\begin{array}{c}3 \\
(0.9)\end{array}$ & $\begin{array}{c}3 \\
(0.9)\end{array}$ & 321 & 321 \\
\hline
\end{tabular}

DWI = diffusion-weighted imaging 

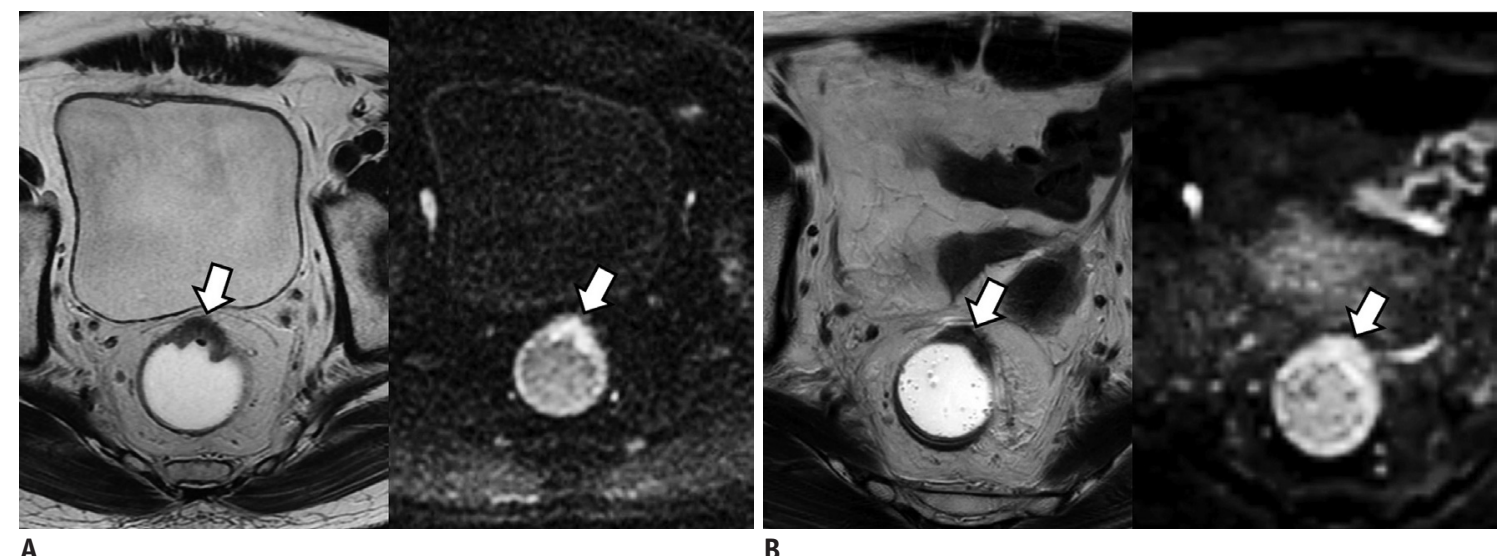

B

Fig. 4. Cases showing usefulness of DWI for assessment of mrTRG in 71-year-old man with rectal cancer.

A. On T2W axial (left) and DWI (right) before CRT, tumor (arrow) is located between 11 and 1 o'clock on rectum. Tumor shows intermediate high SI on T2WI and diffusion restriction on DWI. B. On T2WI (left) obtained after CRT, most of tumor (arrow) was replaced by fibrosis with dark SI. Therefore, two radiologists reported mrTRG as grade 2. However, on post-CRT DWI (right), tumor (arrow) still shows high SI suggesting presence of viable tumors. Two radiologists changed mrTRG into grade 3. On histopathology, there were intermingled tumor cells within fibrosis (not shown). Pathologists finally graded PTRG as grade 3. T2WI $=$ T2-weighted image
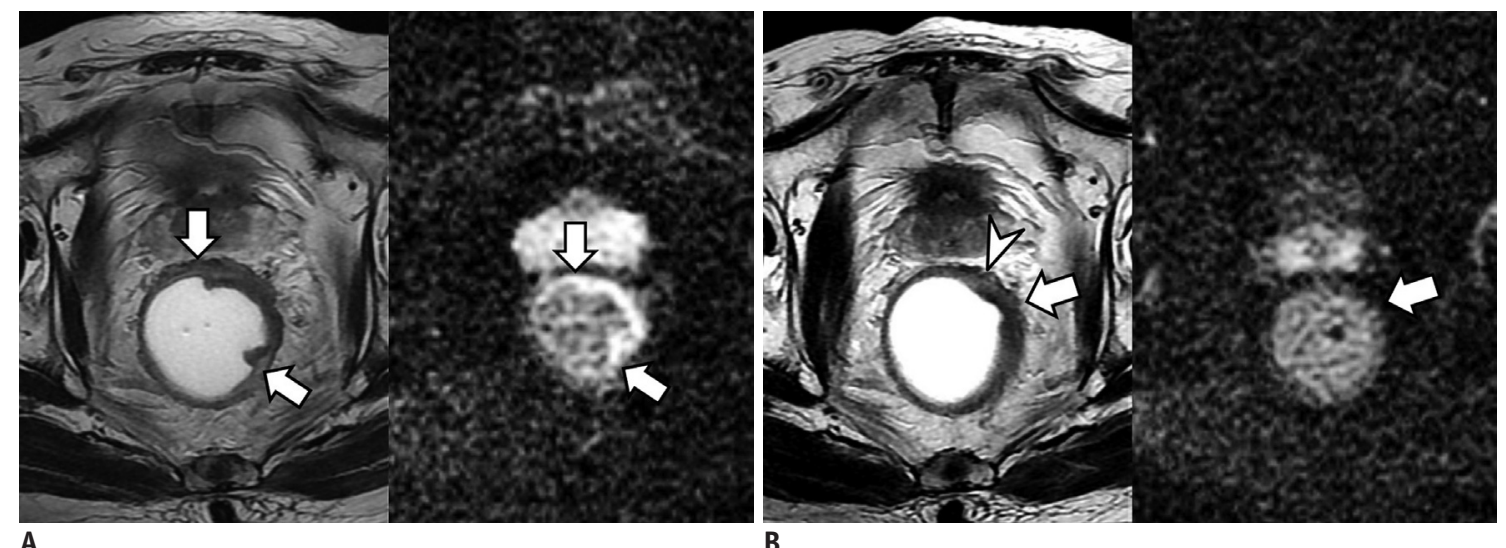

Fig. 5. Cases showing usefulness of DWI for assessment of mrTRG in 80-year-old man with rectal cancer.

A. On T2W axial (left) and DWI (right) before CRT, tumor (arrows) is located between 12 and 5 o'clock position of rectum. Tumor shows intermediate high SI on T2WI (left) and diffusion restriction on DWI (right). B. After CRT, most of tumor (arrow) was replaced by fibrosis with dark SI on T2WI (left). However, there is suspicious area (arrowhead) with intermediate high SI at periphery of tumor. Therefore, two radiologists reported mrTRG as grade 3. However, post-CRT DWI (right) shows completely dark SI at entire tumor (arrow) which suggests absence of viable tumors. Radiologists changed mrTRG into grade 2. On histopathology, there were no tumor cells within fibrosis (not shown). Pathologists finally graded pTRG as grade 2 .

For the association between histopathologic features and DFS, ypT (HR, 5.380; $p<0.001)$, ypN (HR, 3.592, $p<$ $0.001)$, and extramesorectal LN (HR, 8.082; $p<0.001)$ were significantly related with DFS on a univariate analysis. On a multivariate analysis, ypT (HR, 4.832; $p<0.001)$, and extramesorectal LN $(H R, 5.496 ; p<0.001)$ remained as significant factors and ypN (HR, 1.893; $p=0.061)$ had a borderline significance related to DFS.

Based on a 5-point grading system, DFS was significantly different among the mrTRG groups but not on pTRG. The 5-year DFS rate was $100 \%$ for mrTRG $1,85.6 \%$ for mrTRG $2,85.8 \%$ for mrTRG $3,80.3 \%$ for mrTRG 4 , and $62.5 \%$ for
$\operatorname{mrTRG} 5(p=0.039)$. However, the 5 -year DFS rates were not significantly different among the five pTRG groups (83.3\% for pTRG 1,91.8\% for PTRG 2, 85.7\% for PTRG 3, $72.5 \%$ for pTRG 4 , and no event for pTRG 5) $(p=0.072)$. By using a binomial classification, DFS was significantly different between the mrTRG groups $(p=0.036)$ and between the pTRG groups $(p=0.018)$. For mrTRG, the 5 -year DFS rate was $88.3 \%$ for good responders and $79.5 \%$ for poor responders $(p=0.036)$. For pTRG, the 5 -year DFS rate was $86.0 \%$ for good responders and $73.4 \%$ for poor responders $(p=0.018)$. Survival curves of five mrTRG and pTRG groups regarding DFS are presented in Figure 7. 
Table 5. Results of Univariate and Multivariate Analysis of MRI Features for Overall Survival and Disease-Free Survival

\begin{tabular}{|c|c|c|c|c|c|c|c|}
\hline \multirow{2}{*}{ MR Features } & \multirow{2}{*}{ Values } & \multicolumn{3}{|c|}{ Univariate Analysis } & \multicolumn{3}{|c|}{ Multivariate Analysis } \\
\hline & & HR & $95 \% \mathrm{CI}$ & $P$ & $\mathrm{HR}$ & $95 \% \mathrm{CI}$ & $P$ \\
\hline \multicolumn{8}{|l|}{ Overall survival } \\
\hline \multicolumn{8}{|l|}{ Post-CRT MRI } \\
\hline ycN category & & 2.295 & $1.281-4.114$ & 0.005 & & & \\
\hline Negative & 240 & & & & & & \\
\hline Positive & 81 & & & & & & \\
\hline EMVI $_{\text {post }}$ & & 3.314 & $1.547-7.101$ & 0.002 & 2.259 & $1.006-3.218$ & 0.048 \\
\hline Negative & 294 & & & & & & \\
\hline Positive & 27 & & & & & & \\
\hline$E M D_{\text {post }}$ & & 1.082 & $1.015-1.153$ & 0.016 & & & \\
\hline Mean & 2.38 & & & & & & \\
\hline SD & 3.39 & & & & & & \\
\hline \multicolumn{8}{|l|}{ mrTRG } \\
\hline 5-point grading & & 6.615 & $1.188-31.993$ & 0.024 & & & \\
\hline Binomial classification & & 2.172 & $1.205-3.913$ & 0.010 & & & \\
\hline \multicolumn{8}{|l|}{ Disease-free survival } \\
\hline \multicolumn{8}{|l|}{ Pre-CRT MRI } \\
\hline EMVI $_{\text {pre }}$ & & 3.120 & $1.605-6.068$ & 0.001 & & & \\
\hline Negative & 279 & & & & & & \\
\hline Positive & 42 & & & & & & \\
\hline \multicolumn{8}{|l|}{ Post-CRT MRI } \\
\hline ycT category* & & 2.148 & $1.161-3.973$ & 0.015 & & & \\
\hline Good* & 166 & & & & & & \\
\hline Poor & 155 & & & & & & \\
\hline ycN category & & 2.501 & $1.369-4.569$ & 0.003 & & & \\
\hline Negative & 240 & & & & & & \\
\hline Positive & 81 & & & & & & \\
\hline Extramesorectal $\mathrm{LN}_{\text {post }}$ & & 3.273 & $1.650-6.491$ & 0.001 & 2.610 & $1.290-5.281$ & 0.008 \\
\hline Negative & 285 & & & & & & \\
\hline Positive & 36 & & & & & & \\
\hline EMVI $_{\text {post }}$ & & 5.879 & $2.947-11.727$ & $<0.001$ & 5.011 & $2.463-10.195$ & $<0.001$ \\
\hline Negative & 422 & & & & & & \\
\hline Positive & 50 & & & & & & \\
\hline$E M D_{\text {post }}$ & & 1.111 & $1.045-1.181$ & 0.001 & & & \\
\hline Mean & 2.772 & & & & & & \\
\hline SD & 3.826 & & & & & & \\
\hline \multicolumn{8}{|l|}{ mrTRG } \\
\hline 5-point grading & & 7.974 & $1.445-44.015$ & 0.039 & & & \\
\hline Binomial classification & & 1.917 & $1.044-3.522$ & 0.036 & & & \\
\hline
\end{tabular}

*Tumors are graded into two groups (good and poor responders) according to ypT category. ypT1, T2, T3a are classified as good responders and ypT3b, T3c, T3d, and T4 as poor responders. $\mathrm{CI}=$ confidence interval, HR = hazard ratio

\section{DISCUSSION}

Our study showed that mrTRG based on both a 5-point grading system and a binomial classification can be used as a significant imaging biomarker in predicting patients' prognosis in terms of OS and DFS. More specifically, a 5-year OS rate was significantly different among the five mrTRG groups $(100 \%$ for $\operatorname{mrTRG} 1,92.7 \%$ for $\operatorname{mrTRG} 2,89.6 \%$ for mrTRG 3, $80.0 \%$ for mrTRG 4 , and $40.0 \%$ for mrTRG 5 , respectively) ( $p=0.024)$ and between the two mrTRG groups $(91.0 \%$ for good responders and $79.1 \%$ for poor responders) $(p=0.010)$. Considering that reassessment of the tumor after neoadjuvant treatment is essential to enable optimized treatment for patients with locally 


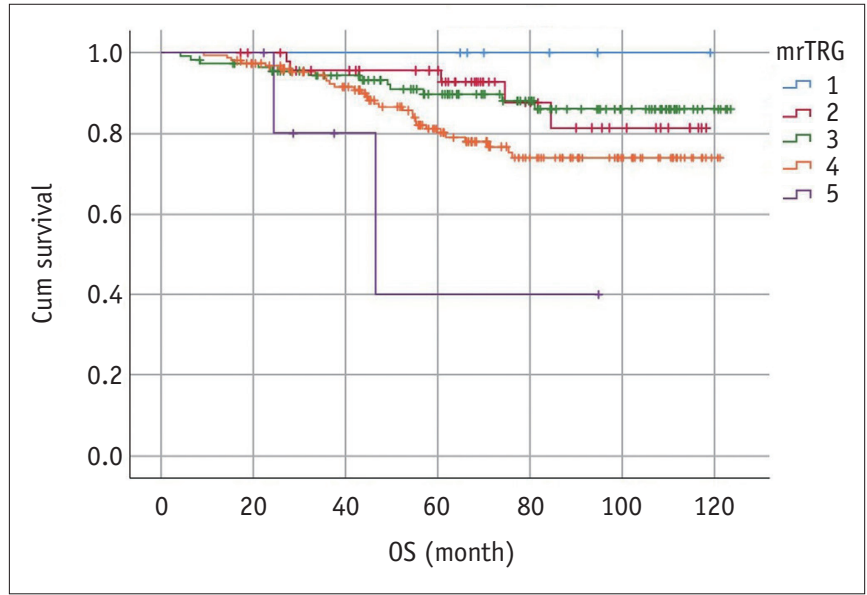

A

Fig. 6. OS curves according to TRG on MRI (A) and pathology (B) based on 5-point grading system. OS rate was significantly different among mrTRG groups $(p=0.024)$ and pTRG groups $(p=0.038)$. Specifically, 5 -year survival rate was $100 \%$ for $\operatorname{mrTRG} 1,92.7 \%$ for $\mathrm{mrTRG} 2$, $89.6 \%$ for mrTRG 3, 80.1\% for mrTRG 4, and 40.0\% for mrTRG 5. However, it was $85.7 \%$ for pTRG 1, 97.8\% for pTRG 2, 84.3\% for pTRG 3, $74.9 \%$ for pTRG 4, and $100 \%$ for pTRG 5. Cum survival = cumulative survival, $0 \mathrm{~S}=$ overall survival

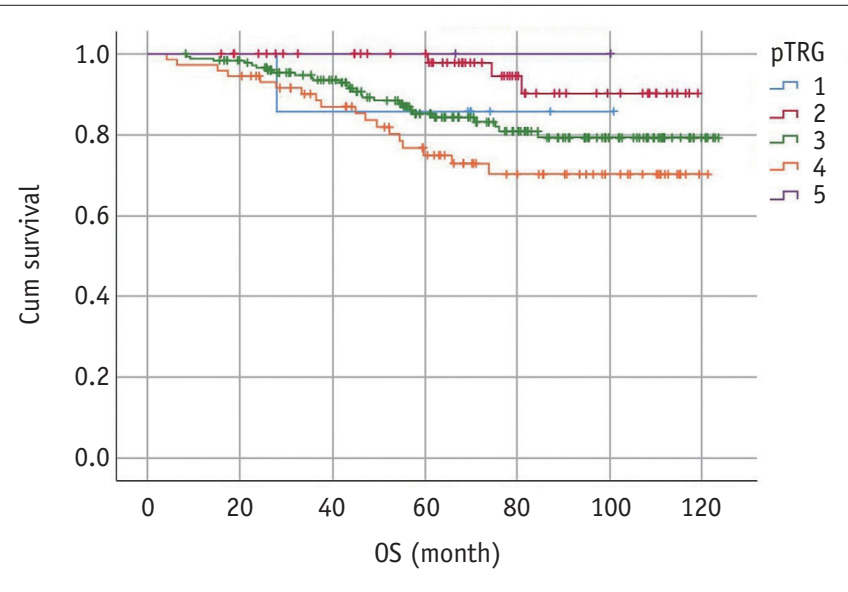

B

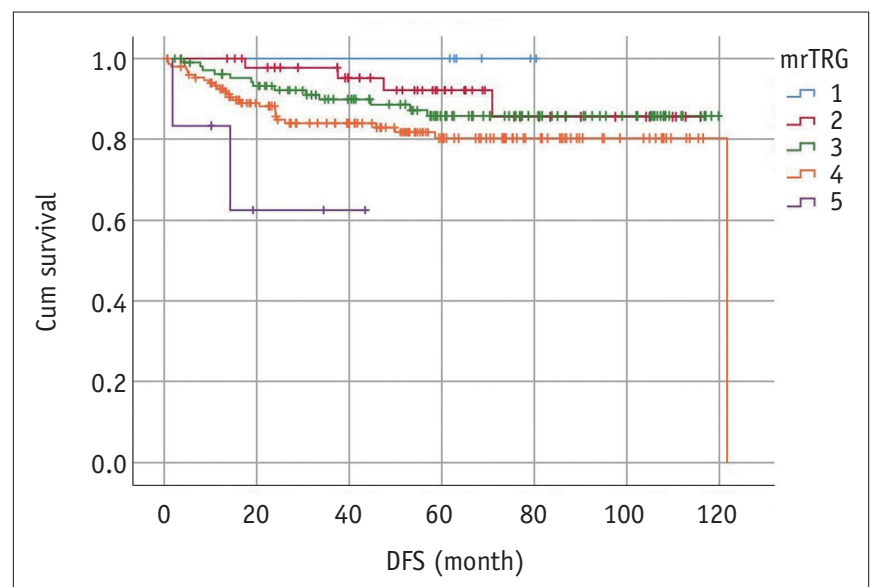

A

Fig. 7. DFS curves according to TRG on MRI (A) and pathology (B) based on 5-point grading system. Specifically, 5-year DFS rate was $100 \%$ (no event) for mrTRG 1, 85.6\% for mrTRG 2, 85.8\% for mrTRG 3, 80.3\% for mrTRG 4, and 62.5\% for mrTRG 5 ( $p=0.039$ ). 5-year DFS rates were not significantly different among five pTRG groups (83.3\% for pTRG 1,91.8\% for pTRG 2, 85.7\% for pTRG 3, 72.5\% for pTRG 4, and no event for pTRG 5$)(p=0.072)$. Cum survival = cumulative survival, DFS = disease-free survival

advanced rectal cancer $(3,27,28)$, our results seem to be promising. We thought that technological development such as high-resolution imaging in MRI and accumulated knowledge in rectal MRI to discriminate cellular component of tumor signal from fibrosis might attribute to this excellent performance of mrTRG. Particularly in the clinical setting, the subgroup of patients with unfavorable posttreatment MRI features appears to be at a higher risk of local or systemic recurrence following a standard total mesorectal excision. Therefore, clinicians including rectal surgeons may be warned of this information preoperatively. Furthermore, based on a 5-point grading system, mrTRG outperformed pTRG in assessing patients' prognosis

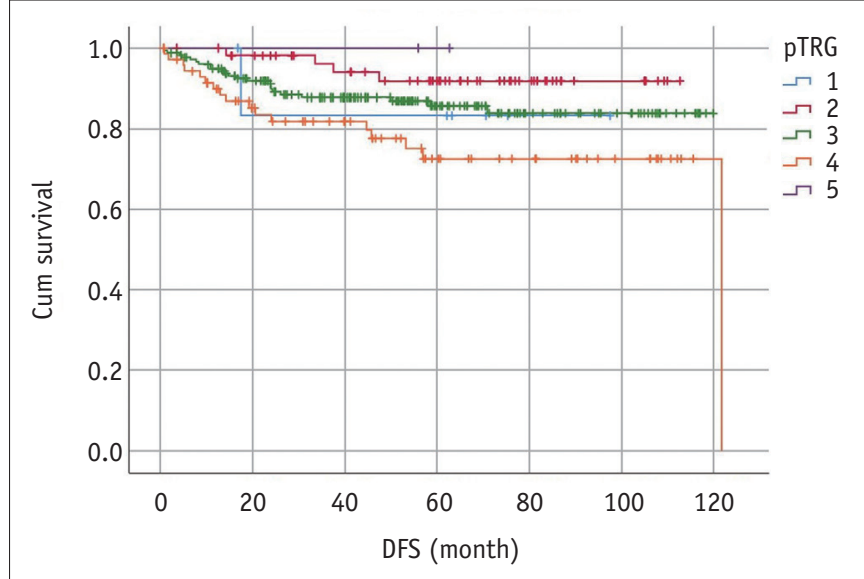

especially for DFS. Even though an interobserver agreement between the pathologists was not analyzed and provided in this study, we heard from a personal communication that poor agreement between the two pathologists was noted (29). Therefore, we speculated that poor agreement between the pathologists might be one of the reasons for an insignificant result of pTRG based on a 5-point grading system in predicting DFS.

In our study, agreement between mrTRG and pTRG based on a 5-point grading system was fair (weighted kappa value $=0.280$ ). We found that a majority of the discrepant cases $(131 / 178,73.6 \%)$ was due to an overestimation of TRG on MRI compared with pathology. In particular, there 
were 85 cases in which mrTRG was 4, while pTRG was 3. Such an overestimation of TRG by MRI might be closely related to the fact that CRT-induced inflammation or edema intermingled with fibrosis may mimic the tumor signal on post-CRT MRI. We believe that such tumor-mimicking post-CRT changes can be differentiated from true tumor signal using DWI. Indeed, when DWI was added during MR interpretation, kappa values between two radiologists was improved from 0.309 to 0.376 on a 5-point grading system as well as from 0.364 to 0.438 on a binomial classification. Furthermore, mean time interval between post-CRT MRI and operation was 48.3 days in our study. Considering that post-CRT surgery is usually performed 6-8 weeks after the completion of CRT, a relatively long time interval between post-CRT MRI and operation may be partially responsible for the weak agreement between mrTRG and pTRG in our study.

We also found that the weighted kappa value for interobserver agreement on mrTRG between the radiologists was 0.309 (fair agreement) in our study, which was lower than that $(0.65$, good agreement) reported by the MERCURY study group (15). This difference may be due to the experience and technical differences in the quality of MR scans. However, when DWI sequence was added to T2WIs, the weighted kappa value was improved to 0.376 (fair agreement). Accordingly, we recommend the use of DWI in assessing mrTRG because DWI can give a supplementary clue to detect viable residual tumor cells in addition to T2 signal changes.

Based on multivariate analysis, only post-CRT MRI features such as $\mathrm{EMVI}_{\text {post }}(H R, 2.259 ; p=0.048)$ for $0 \mathrm{~S}$ and extramesorectal LN (HR, 2.610; $p=0.008)$ and EMVI post (HR, 5.011; $p<0.001)$ for DFS were the significant MR parameters in predicting patients' prognosis. These observations were well in line with those of the previous studies (30) and our common belief. This is likely because treatment response to CRT may vary among patients, and it is generally difficult to predict the responsiveness before treatment.

Even though post-treatment assessment has focused on demonstrating pathological complete response ( $P C R)$ in clinical trials, $\mathrm{pCR}$ might be a controversial endpoint due to potential under-sampling of surgical specimens despite the use of a predefined protocol. Actually, identifying only pCR may underestimate the benefit of neoadjuvant therapy because it is well known that patients showing a good response also have equally good outcomes as patients with $\mathrm{pCR}$. Therefore, recent investigations have focused on distinguishing good from poor responders rather than defining $\mathrm{PCR}$. In this context, we thought that the good concordance rate $(62.3 \%, 200 / 321)$ between mrTRG and pTRG based on a binomial classification shown in this study was an encouraging result. Such a good concordance rate may alleviate concerns about evaluating mrTRG in post-CRT cases, where there may be CRT-related edema and inflammation.

Our study has several limitations. First, although we performed a large-scale analysis enrolling 321 patients, this single-center study can provide deviated raw data regarding limited distribution in geographic and demographic aspects. In addition, although mrTRG was determined to be a significant variable for predicting $0 \mathrm{~S}$ and DFS on univariate analysis, it was not included as a significant factor on multivariate analysis. However, considering high HRs of mrTRG on univariate analysis (6.165 for OS and 7.974 for DFS) which strongly suggest the significance of mrTRG, relatively small numbers of total study population and event cases may be partially responsible for such insignificant result of mrTRG on multivariate analysis. Therefore, multicenter studies enrolling a sufficiently large number of patients are strongly warranted to validate the prognostic value of mrTRG. Second, even though we speculated that an insignificant result of pTRG based on a 5-point grading system in predicting DFS may be related to a poor agreement between the pathologists, we did not assess interobserver agreement between pathologists for evaluating PTRG. Finally, given that SI on DWI can be affected by a field strength of MRI (1.5T vs. 3T), radiologists' performance can be influenced by the field strength of MRI. However, we did not analyze the effect of field strength on interobserver agreement for mrTRG.

In conclusion, compared with pTRG, mrTRG based on a 5-point scoring system predicts patients' prognosis with comparable performance on OS and DFS. Furthermore, the addition of DWI on T2-weighted MRI may improve the interobserver agreement on mrTRG. Therefore, MRI with DWI can be useful in predicting prognosis in patients with locally advanced rectal cancer.

\section{Supplementary Materials}

The Data Supplement is available with this article at https://doi.org/10.3348/kjr.2019.0797.

\section{Conflicts of Interest}

J.H.Y. received consultancy fees from Samsung Electronics. 
But the author's activities not related to the present article.

ORCID iDs

Se Hyung Kim

https://orcid.org/0000-0001-8664-0356

Heera Yoen

https://orcid.org/0000-0001-5583-6065

Hye Eun Park

https://orcid.org/0000-0003-1048-2827

Jeong Hee Yoon

https://orcid.org/0000-0002-9925-9973

Bo Yun Hur

https://orcid.org/0000-0001-6549-6076

Jae Seok Bae

https://orcid.org/0000-0003-2768-7917

Jung Ho Kim

https://orcid.org/0000-0002-6031-3629

Joon Koo Han

https://orcid.org/0000-0001-5916-5545

\section{REFERENCES}

1. Kapiteijn E, Marijnen CA, Nagtegaal ID, Putter H, Steup WH, Wiggers $\mathrm{T}$, et al. Preoperative radiotherapy combined with total mesorectal excision for resectable rectal cancer. $N$ Engl J Med 2001;345:638-646

2. Capirci C, Valentini V, Cionini L, De Paoli A, Rodel C, GlynneJones $R$, et al. Prognostic value of pathologic complete response after neoadjuvant therapy in locally advanced rectal cancer: long-term analysis of $566 \mathrm{ypCR}$ patients. Int J Radiat Oncol Biol Phys 2008;72:99-107

3. Park IJ, You YN, Agarwal A, Skibber JM, Rodriguez-Bigas MA, Eng $C$, et al. Neoadjuvant treatment response as an early response indicator for patients with rectal cancer. $\mathrm{J}$ Clin Oncol 2012;30:1770-1776

4. Rödel C, Martus $P$, Papadoupolos T, Füzesi L, Klimpfinger M, Fietkau $R$, et al. Prognostic significance of tumor regression after preoperative chemoradiotherapy for rectal cancer. $J$ Clin Oncol 2005;23:8688-8696

5. Maas M, Nelemans PJ, Valentini V, Das P, Rödel C, Kuo LJ, et al. Long-term outcome in patients with a pathological complete response after chemoradiation for rectal cancer: a pooled analysis of individual patient data. Lancet Oncol 2010;11:835-844

6. Dworak 0, Keilholz L, Hoffmann A. Pathological features of rectal cancer after preoperative radiochemotherapy. Int J Colorectal Dis 1997;12:19-23

7. Wheeler JM, Warren BF, Mortensen NJ, Ekanyaka N, Kulacoglu $H$, Jones $A C$, et al. Quantification of histologic regression of rectal cancer after irradiation: a proposal for a modified staging system. Dis Colon Rectum 2002;45:1051-1056
8. Vecchio FM, Valentini V, Minsky BD, Padula GD, Venkatraman ES, Balducci M, et al. The relationship of pathologic tumor regression grade (TRG) and outcomes after preoperative therapy in rectal cancer. Int J Radiat Oncol Biol Phys 2005;62:752-760

9. Seo N, Kim H, Cho MS, Lim JS. Response assessment with MRI after chemoradiotherapy in rectal cancer: current evidences. Korean J Radiol 2019;20:1003-1018

10. Patel UB, Brown G, Rutten H, West N, Sebag-Montefiore D, Glynne-Jones R, et al. Comparison of magnetic resonance imaging and histopathological response to chemoradiotherapy in locally advanced rectal cancer. Ann Surg Oncol 2012;19:2842-2852

11. Kim YH, Kim DY, Kim TH, Jung KH, Chang HJ, Jeong SY, et al. Usefulness of magnetic resonance volumetric evaluation in predicting response to preoperative concurrent chemoradiotherapy in patients with resectable rectal cancer. Int J Radiat Oncol Biol Phys 2005;62:761-768

12. Kang JH, Kim YC, Kim H, Kim YW, Hur H, Kim JS, et al. Tumor volume changes assessed by three-dimensional magnetic resonance volumetry in rectal cancer patients after preoperative chemoradiation: the impact of the volume reduction ratio on the prediction of pathologic complete response. Int J Radiat Oncol Biol Phys 2010;76:1018-1025

13. Yeo SG, Kim DY, Park JW, Oh JH, Kim SY, Chang HJ, et al. Tumor volume reduction rate after preoperative chemoradiotherapy as a prognostic factor in locally advanced rectal cancer. Int J Radiat Oncol Biol Phys 2012;82:e193-e199

14. Xiao J, Tan Y, Li W, Gong J, Zhou Z, Huang Y, et al. Tumor volume reduction rate is superior to RECIST for predicting the pathological response of rectal cancer treated with neoadjuvant chemoradiation: results from a prospective study. Oncol Lett 2015;9:2680-2686

15. Patel UB, Taylor F, Blomqvist L, George C, Evans H, Tekkis P, et al. Magnetic resonance imaging-detected tumor response for locally advanced rectal cancer predicts survival outcomes: MERCURY experience. J Clin Oncol 2011;29:3753-3760

16. Salerno G, Chandler I, Wotherspoon A, Thomas K, Moran B, Brown $G$. Sites of surgical wasting in the abdominoperineal specimen. Br J Surg 2008;95:1147-1154

17. Siddiqui MR, Gormly KL, Bhoday J, Balyansikova S, Battersby $\mathrm{NJ}$, Chand M, et al. Interobserver agreement of radiologists assessing the response of rectal cancers to preoperative chemoradiation using the MRI tumour regression grading (mrTRG). Clin Radiol 2016;71:854-862

18. Sclafani F, Brown G, Cunningham D, Wotherspoon A, Mendes LST, Balyasnikova $S$, et al. Comparison between MRI and pathology in the assessment of tumour regression grade in rectal cancer. Br J Cancer 2017;117:1478-1485

19. Kim SH, Lee JM, Hong SH, Kim GH, Lee JY, Han JK, et al. Locally advanced rectal cancer: added value of diffusionweighted MR imaging in the evaluation of tumor response to neoadjuvant chemo- and radiation therapy. Radiology 2009;253:116-125 
20. Elmi A, Hedgire SS, Covarrubias D, Abtahi SM, Hahn PF, Harisinghani M. Apparent diffusion coefficient as a noninvasive predictor of treatment response and recurrence in locally advanced rectal cancer. Clin Radiol 2013;68:e524-e531

21. Moon SJ, Cho SH, Kim GC, Kim WH, Kim HJ, Shin KM, et al. Complementary value of pre-treatment apparent diffusion coefficient in rectal cancer for predicting tumor recurrence. Abdom Radiol (NY) 2016;41:1237-1244

22. Sassen S, de Booij M, Sosef M, Berendsen R, Lammering G, Clarijs $R$, et al. Locally advanced rectal cancer: is diffusion weighted MRI helpful for the identification of complete responders (ypTONO) after neoadjuvant chemoradiation therapy? Eur Radiol 2013;23:3440-3449

23. Quirke P, Durdey P, Dixon MF, Williams NS. Local recurrence of rectal adenocarcinoma due to inadequate surgical resection. Histopathological study of lateral tumour spread and surgical excision. Lancet 1986;2:996-999

24. Quirke P, Dixon MF. The prediction of local recurrence in rectal adenocarcinoma by histopathological examination. Int J Colorectal Dis 1988;3:127-131

25. Mandard AM, Dalibard F, Mandard JC, Marnay J, HenryAmar M, Petiot JF, et al. Pathologic assessment of tumor regression after preoperative chemoradiotherapy of esophageal carcinoma. Clinicopathologic correlations. Cancer
$1994 ; 73: 2680-2686$

26. Willett CG, Badizadegan K, Ancukiewicz M, Shellito PC. Prognostic factors in stage T3NO rectal cancer: do all patients require postoperative pelvic irradiation and chemotherapy? Dis Colon Rectum 1999;42:167-173

27. Lee SY, Kim CH, Kim YJ, Kim HR. Oncologic outcomes according to the treatment strategy in radiologic complete responders after neoadjuvant chemoradiation for rectal cancer. Oncology 2015;89:311-318

28. Jang JK, Lee JL, Park SH, Park HJ, Park IJ, Kim JH, et al. Magnetic resonance tumour regression grade and pathological correlates in patients with rectal cancer. $\mathrm{Br} J$ Surg 2018;105:1671-1679

29. Chetty R, Gill P, Govender D, Bateman A, Chang HJ, Deshpande $V$, et al. International study group on rectal cancer regression grading: interobserver variability with commonly used regression grading systems. Hum Pathol 2012;43:19171923

30. Lee ES, Kim MJ, Park SC, Hur BY, Hyun JH, Chang HJ, et al. Magnetic resonance imaging-detected extramural venous invasion in rectal cancer before and after preoperative chemoradiotherapy: diagnostic performance and prognostic significance. Eur Radiol 2018;28:496-505 